\title{
Reasons for discordances between ultrasonography and magnetic resonance imaging in the evaluation of the ankle, hindfoot and heel of the patients with rheumatoid arthritis
}

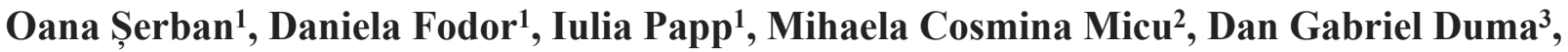 \\ Csaba Csutak ${ }^{3}$, Manuela Lenghel ${ }^{3}$, Maria Bădărînză ${ }^{1}$, Adriana Albu ${ }^{1}$
}

${ }^{1} 2^{\text {nd }}$ Internal Medicine Department, "Iuliu Hatieganu" University of Medicine and Pharmacy, ${ }^{2}$ Rheumatology Division, Rehabilitation Clinical Hospital, ${ }^{3}$ Radiology Department, "Iuliu Hatieganu" University of Medicine and Pharmacy, Cluj-Napoca, Romania

\begin{abstract}
Aim: To compare the ultrasonography (US) performance with magnetic resonance imaging (MRI) in identifying pathology in ankles, hindfeet and heels of rheumatoid arthritis (RA) patients and to evaluate the reasons for discordances between the two imaging methods. Material and methods: RA patients were enrolled and evaluated using the Clinical Disease Activity Index (CDAI) and the Disease Activity Score 28 with C-reactive Protein (DAS28-CRP). The ankle (tibiotalar joint, tendons), hindfoot (talonavicular, subtalar joints) and heel of the most symptomatic or dominant foot (for the asymptomatic patient) were evaluated by two pairs of examiners using US and contrast-enhanced MRI. Results: Totally, 105 joints, 245 tendons and 35 heels in 35 patients [mean age $59.2 \pm 11.25$ years old, median disease duration 36 (16.5-114), mean CDAI 19.87 \pm 12.7 ] were evaluated. The interobserver agreements between the two sonographers, and the two radiologists were good and very good $(\mathrm{k}=0.624-0.940)$. The overall agreement between US and MRI was very good for subcalcaneal panniculitis $(\mathrm{k}=0.928$, $\mathrm{p}<0.001)$, moderate for synovitis $(\mathrm{k}=0.463, \mathrm{p}<0.001)$ and tenosynovitis $(\mathrm{k}=0.514, \mathrm{p}<0.001)$, fair for osteophytes $(\mathrm{k}=0.260$, $\mathrm{p}=0.004)$, and poor for erosions $(\mathrm{k}=0.063, \mathrm{p}=0.308)$ and heel's structures. MRI found more erosions, synovitis, osteophytes, tenosynovitis and retrocalcaneal bursitis, but US found more enthesophytes and plantar fasciitis. Many of the discordances between the two imaging techniques have explanations related to the technique itself or definition of the pathologic findings. Conclusions: US is comparable to MRI for the evaluation of ankle, hindfoot and heel in RA patients and discordances in the interpretation of the pathological findings/normal structures must be carefully analyzed.
\end{abstract}

Keywords: ankle; hindfoot; heel; rheumatoid arthritis; ultrasound; MRI

\section{Introduction}

Rheumatoid arthritis (RA) is a chronic inflammatory disease that usually affects symmetrically multiple joints. Apart from serological and clinical criteria, the imaging

Received 02.10.2019 Accepted 10.11.2019

Med Ultrason

2019, Vol. 21, No 4, 405-413

Corresponding author: Daniela Fodor, $\mathrm{MD}, \mathrm{PhD}$

$2^{\text {nd }}$ Internal Medicine Department

"Iuliu Hatieganu" University of Medicine and

Pharmacy, Cluj-Napoca, Romania

2-4 Clinicilor street,

400006 Cluj-Napoca, Romania

E-mail: dfodor@umfcluj.ro techniques increase the diagnosis sensitivity and have a substantial role in RA monitoring [1]. The inflammatory (synovitis, tenosynovitis, bursitis, bone marrow edema) and structural damage (bone erosions) modifications are better evaluated and quantified using X-rays, ultrasound (US) or magnetic resonance imaging (MRI) compared with clinical examination [2-4]. X-rays are considered to be the initial imaging technique used to detect damage, but US, and especially MRI, are able to recognize the erosions in earlier stages [1]. For synovitis or tenosynovitis identification, MRI and US are the preferred imaging techniques.

The US definitions and grading for synovitis, tenosynovitis, erosions, osteophytes, enthesophytes, retrocal- 
caneal bursitis and plantar fasciitis are well established by the European League Against Rheumatism/Outcome Measures in Rheumatology Clinical Trials (EULAR/ OMERACT) working groups [5-10], as well as the standardized US examination technique for each musculoskeletal region [11]. The MRI-OMERACT group elaborated MRI definitions for joint synovitis, tenosynovitis, bone erosions and bone edema [12] and developed a scoring system for the evaluation of inflammation and damage of the hand and wrist in RA patients - RA MRI scoring system (RAMRIS) [13]. Comparative studies of MRI with US findings in RA patients related to hand and wrist synovitis [14,15], including subclinical [2], erosions [3] and tenosynovitis were published [15]. Though the interobserver agreements for US examinations were moderate/ good, the agreement between MRI and US was mostly moderate. There are studies that found lower sensitivity for MRI in identifying small joints synovitis compared with grey-scale (GS) or power Doppler (PD) US [14], while others found the opposite $[2,15]$. Only a few studies compared the results of the two techniques in ankles $[16,17]$ and hindfeet [18], but no comparison was made for the heel's structures (apart from the heel's fat pad involvement [19]). The involvement of the feet and ankles is early in RA and leads in time to architectural deformities and structural damage, resulting in significant foot disability and impairment of the quality of life [20] and, for this reason, these regions have to receive the same attention as the wrists and hands.

Some of the factors that contribute to the differences in results obtained in MRI and US examinations are understandable and predictable, as machine performances and settings, examination protocol, interobserver/intraobserver variability. Other factors are possibly related to the pathology definitions used by each technique, the lack of a clear consensus for the normality status or the ability to better identify a particular type of abnormality. But these discordances could have direct effects on a medical judgment and patient's management.

The aim of the study was to evaluate US performances in identifying ankle, hindfoot and heel pathological findings in patient with RA compared with MRI, and to analyze the reasons that could explain the discordant interpretations of the same lesion/aspect of an anatomical structure by the two imaging methods.

\section{Material and methods}

\section{Patients}

Between April and December 2018, consecutive patients with RA presenting in our day-hospital clinic were enrolled. The diagnosis of RA according to the
2010 American College of Rheumatology (ACR)/EULAR classification criteria [21] and age over 18 years old were the inclusion criteria. The exclusion criteria were contraindications for MRI examination (e.g. pacemakers, MRI-unfriendly prostheses or devices). Each patient signed a written consent before enrolment and approval of the Ethics Committee of the University was obtained.

A rheumatologist with 4-years of experience in rheumatology (IP) collected the demographical data and history for each patient, performed the physical examination, counted the tender and swollen joints and established the disease activity calculating Clinical Disease Activity Index (CDAI) and Disease Activity Score 28 with C-reactive Protein (DAS28-CRP).

For further evaluation we chose the most symptomatic foot or the dominant foot in asymptomatic patients.

\section{Ultrasound}

The ultrasound evaluation was performed using a GE Logiq 7 ultrasound machine (GE Healthcare, Chicago, USA) with ML6-15 transducer. The frequency and the focus were set according to the structure of interest. For PDUS, the frequency was set to $9 \mathrm{MHz}$, the color box was large enough to include entirely the region of interest and the upper margin of the image, the gain was set as to obtain maximal blood flow with minimal noise, and the pulse repetition frequency (PRF) to $800 \mathrm{MHz}$. The US examination was performed by two sonographers (with 5 and 20-years of experience in musculoskeletal US), in the same day, blinded for each other's results, and images for each assessed structure were stored. The disagreements between the two examiners were resolved by a third examiner with more than 15 -years of experience in musculoskeletal US.

The examination was realized with the patient in a supine position, with the knee flexed $90^{\circ}$ and the sole resting on the bed for ankle, hindfoot joints and tendons. For the heel's structures the patients was in a prone position with the foot hanging at the edge of the bed in a neutral position. Based on a preestablished protocol and according to EULAR-OMERACT recommendations, definitions and grading [5-11,22,23], tibiotalar (TT), talonavicular (TN) and subtalar (ST) joints synovitis, erosions and osteophytes were assessed, as well as tibialis anterior (TA), extensor hallucis longus (EHL), extensor digitorum communis (EDC), tibialis posterior (TP), flexor digitorum communis (FDC), flexor hallucis longus (FHL) and peroneal tendons tenosynovitis. In the heel region, the Achille's tendon, the presence of enthesophytes, retrocalcaneal bursitis, posterior and inferior calcaneal erosions, plantar fasciitis, perifasciitis and subcalcaneal panniculitis were evaluated. 
We considered the tibiotalar joint (TT) for the ankle region, talonavicular joint (TN) and subtalar joint (ST) for the hindfoot region and Achille's tendon and plantar fascia with their surrounding structures for the heel's region.

MRI

All the examinations were performed on a 1.5 T MRI equipment (Signa Explorer, GE Healthcare, France). The patient was placed in supine position with the foot pointing towards the magnet (feet first supine), with the ankle flexed at $90^{\circ}$ and the knee extended. The ankle coil (HD T/R Knee/Foot Coil by Invivo) was placed over the foot and ankle and locked properly. The laser beam localizer was centered over the hindfoot (approximate head of the talus). The image protocol for the ankle, hindfoot and heel comprised the following sequences: sagittal T1-weighted fast spin echo (FSE) with a FoV of 240x260 mm, Matrix size 320x224, slice thickness $3 \mathrm{~mm}$ (TR $450 \mathrm{~ms}$, TE $9 \mathrm{~ms}$ ), sagittal T1-weighted fast spin echo with fat saturated (FS) with a FoV of 240x260 mm, Matrix size 320x224, slice thickness $3 \mathrm{~mm}$ (TR $560 \mathrm{~ms}$, TE $9 \mathrm{~ms}$ ), coronal STIR-sequence with a FoV of 180x180 mm, Matrix size 256x160, slice thickness $3 \mathrm{~mm}$ (TR $2314 \mathrm{~ms}$, TE $43 \mathrm{~ms}$, TI $150 \mathrm{~ms}$ ), axial STIR-sequence with a FoV of 260x210 mm, matrix size 192x152, slice thickness $3 \mathrm{~mm}$ (TR $3017 \mathrm{~ms}$, TE 46,5 ms, TI $150 \mathrm{~ms}$ ), coronal 3 dimensional T1-weighted fast spoiled gradient echo (FSPGR) sequence with a FoV of $180 \times 108 \mathrm{~mm}$, matrix size $192 \times 192$, slice thickness $1 \mathrm{~mm}$ (TR 6,8 ms, TE 3,2 ms) prior to and after intravenous injection of contrast material $[0.2 \mathrm{ml} / \mathrm{kg}$ bodyweight of Gd-DTPA (Omniscan)]. The three-dimensional (3D) T1-weighted FSPGR sequences of the ankle were additionally reconstructed in axial and sagittal planes.

The images were analyses and graded according to the OMERACT RAMRIS system model adapted for the evaluation of the ankle and hindfoot $[13,24,25]$. The pathological changes were assessed at 3 joints level (TT, TN and ST) and 7 tendons level (TA, EHL, EDC, TP, FDC, FHL and peroneal tendons), but also at the level of intertarsian joints (quantified as present/absent). The evaluation of the heel included the identification of Achille's tendon enthesophytes, the retrocalcaneal bursitis, the posterior and inferior calcaneal erosions, subcalcaneal panniculitis and the measurement of the plantar fascia (insagittal T1 images). All MRI studies were interpreted separately by two musculoskeletal radiologists with more than 10-years of experience, blinded for each other's results. The discordance between the two radiologists was solved by a third radiologist with more than 10-years of experience.

The US and MRI definitions of the evaluated abnormalities and their quantifications are detailed in supplementary Table I, on the journal site.

\section{Statistical analysis}

The interobserver agreement between the two examiners for each imaging technique (US and MRI) was measured by calculating Cohen's kappa coefficient (k). The interpretation of the $\mathrm{k}$ coefficient values was as follows: $0-0.20$ poor, $0.20-0.40$ fair, $0.40-0.60$ moderate, $0.60-0.80$ good, and $0.80-1$ very good. The concordance between the two imaging techniques (US and MRI) was measured by calculating the percent of agreement (PA) signifying the number of concordances divided by the number of cases. A PA of less than $40 \%$ was interpreted as poor concordance, $40-60 \%$ as moderate, $60-80 \%$ as good, and more than $80 \%$ as very good. The agreement between US and MRI results was also measured by calculating $\mathrm{k}$ coefficients.

\section{Results}

We enrolled 35 patients with RA, $85.7 \%$ females, mean age 59.2 \pm 11.25 years old, mean BMI $26.5 \pm 5.65$ $\mathrm{kg} / \mathrm{m}^{2}$, median of disease duration 36 (16.5-114) months, mean CDAI 19.87 \pm 12.7 and mean DAS28-CRP 3.5 \pm 1.35 . Totally, 105 joints and 245 tendons were examined.

\section{Interobserver agreement for US and MRI}

The interobserver agreement between the two sonographers and the two radiologists for the ankle, hindfoot and heel's modifications are shown in Table I.

\section{Ultrasound and MRI concordances and \\ discordances}

The overall agreement between US and MRI was moderate for joint synovitis $(\mathrm{k}=0.463, \mathrm{p}<0.001)$ and tenosynovitis $(\mathrm{k}=0.514, \mathrm{p}<0.001)$, poor for erosions ( $\mathrm{k}=0.063$ but non-significant, $\mathrm{p}=0.308)$ and fair for osteophytes $(\mathrm{k}=0.260, \mathrm{p}=0.004)$.

The concordances and discordances between US and MRI at joint / tendon and heel level are shown in Table II, III and IV.

Most discrepancies between US and MRI were observed for erosion. Compared to US, MRI identified more erosions at joints level. In 41/105 joints with erosion on MRI, US findings were normal. Only in $4 / 105$ joints, the erosions identified by US where not described in MRI (all small erosions, in TN and ST joints).

Related to the identification of the ankle and hindfoot synovitis, in 11/105 joints (3 TT, 5 TN and 3 ST joints) US interpreted the pathological findings as synovitis and MRI as intraarticular synovial fluid (fig 1).

Osteophytes were visualized only by MRI in $16 / 105$ joint, and only by US in $5 / 105$ joints

Analyzing the identification of tenosynovitis by each technique, MRI found tenosynovitis when US showed normal tendon sheath aspect in 23/245 tendons, (in all 
Table I. Interobserver agreement for US and MRI evaluation of the ankle, hindfoot and heel in RA patients

\begin{tabular}{|c|c|c|c|}
\hline & & $\begin{array}{l}\text { US } \\
(n=35)\end{array}$ & $\begin{array}{l}\text { MRI } \\
(n=35)\end{array}$ \\
\hline Structure & Parameter & kappa* & kappa* \\
\hline \multicolumn{4}{|l|}{ Joints } \\
\hline \multirow[t]{6}{*}{ TT } & Synovitis & 0.868 & 0.928 \\
\hline & $\mathrm{PD}+$ & 0.842 & - \\
\hline & Synovial fluid & - & 0.867 \\
\hline & Erosions & 0.653 & 0.884 \\
\hline & Bone edema & - & 0.935 \\
\hline & Osteophytes & 0.785 & 0.838 \\
\hline \multirow[t]{6}{*}{$\mathrm{TN}$} & Synovitis & 0.928 & 0.935 \\
\hline & $\mathrm{PD}+$ & 0.822 & - \\
\hline & Synovial fluid & - & 0.881 \\
\hline & Erosions & 0.892 & 0.878 \\
\hline & Bone edema & - & 0.822 \\
\hline & Osteophytes & 0.821 & 0.851 \\
\hline \multirow[t]{6}{*}{ ST } & Synovitis & 0.750 & 0.922 \\
\hline & $\mathrm{PD}+$ & 0.717 & - \\
\hline & Synovial fluid & - & 0.922 \\
\hline & Erosions & 0.842 & 0.884 \\
\hline & Bone edema & - & 0.940 \\
\hline & Osteophytes & 0.639 & 0.892 \\
\hline \multirow[t]{5}{*}{ Intertarsian } & Synovitis & - & 0.868 \\
\hline & Synovial fluid & - & 0.785 \\
\hline & Erosions & - & 0.839 \\
\hline & Bone edema & - & 0.873 \\
\hline & Osteophytes & - & 0.906 \\
\hline Tendons ${ }^{¥}$ & Tenosynovitis & $\begin{array}{c}0.785- \\
0.906^{\S}\end{array}$ & $\begin{array}{r}0.785- \\
0.928\end{array}$ \\
\hline \multirow[t]{4}{*}{ Achille's tendon } & Enthesophytes & 0.915 & 0.658 \\
\hline & Retrocalcaneal bursitis & 0.928 & 0.883 \\
\hline & $\begin{array}{l}\text { Posterior calcaneal } \\
\text { erosions }\end{array}$ & 0.892 & 0.718 \\
\hline & $\begin{array}{l}\text { Posterior calcaneal } \\
\text { bone edema }\end{array}$ & - & 0.785 \\
\hline \multirow[t]{4}{*}{ Plantar fascia } & Plantar fasciitis & 0.878 & 0.624 \\
\hline & Perifasciitis & 0.838 & 0.746 \\
\hline & $\begin{array}{l}\text { Inferior calcaneal } \\
\text { erosions }\end{array}$ & 0.822 & 0.932 \\
\hline & $\begin{array}{l}\text { Inferior calcaneal } \\
\text { bone edema }\end{array}$ & - & 0.839 \\
\hline $\begin{array}{l}\text { Subcalcaneal } \\
\text { panniculitis }\end{array}$ & & 0.838 & 0.922 \\
\hline
\end{tabular}

*all $\mathrm{p}<0.001 ;{ }^{*}$ Ankle’s tendons: TA, EHL, EDL, TP, FDL, FHL and peroneal; ${ }^{\S}$ Except for EDL, FHL, and FDL where $\mathrm{k}=0.653$ $(p<0.001)$; MRI: magnetic resonance imaging; US: ultrasonography; TT: tibiotalar joint; TN: talonavicular joint; ST: subtalar joint; TA: tibialis anterior; EHL: extensor hallucis longus; EDL: extensor digitorum longus; TP: tibialis posterior; FDL: flexor digitorum longus; FHL: flexor hallucis longus.

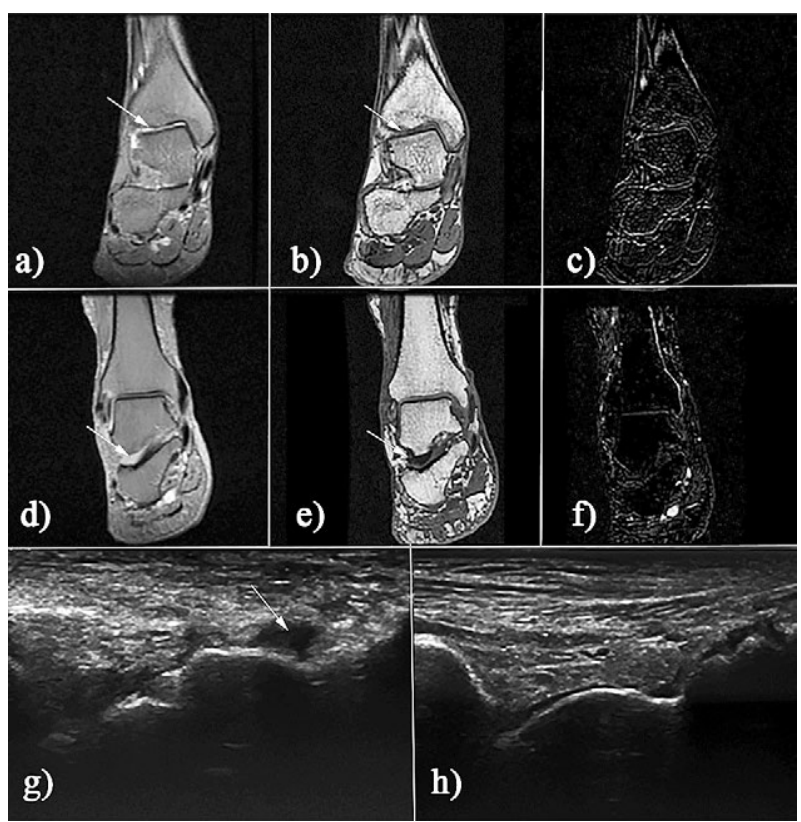

Fig 1. A 52-years old RA patient where MRI showed intraarticular synovial fluid in TT joint: a) coronal STIR sequence showing increased signal intensity without enhancement in b) post-contrast coronal 3D T1 FSPGR and c) post-contrast coronal 3D T1 FSPGR with substraction, and US showed grade 1 synovitis in h) longitudinal scan of the anterior recess of TT joint; A 75-years old RA patients where MRI showed intraarticular synovial fluid in ST joint: d) coronal STIR sequence showing increased signal intensity without enhancement in e) post-contrast coronal 3D T1 FSPGR and f) post-contrast coronal 3D T1 FSPGR with subtraction, and US showed grade 2 synovitis in $\mathrm{g}$ ) lateral scan of the ST joint.

cases grade 1, mild, peritendinous fluid). On the contrary, US found tenosynovitis when MRI showed normal aspect in $3 / 245$ tendons.

In 11/35 cases, US identified Achille's tendon enthesophytes (fig 2), and in 4/35 cases calcaneal erosions (fig 3), none of these being visualized by MRI. The poorest concordances between US and MRI for the evaluation of heel's structures were found for retrocalaneal bursitis and plantar fascia thickness (fig 4). The subcalcaneal panniculitis had the best concordance and agreement between US and MRI (fig 5).

\section{Discussion}

Our study found good and very good interobserver agreement between the two sonographers, and the two radiologists. The concordances of the findings between US and MRI were very good for tenosynovitis and subcalcaneal panniculitis, and good for joints synovitis and osteophytes. Moderate concordances were found for bone erosions (except for calcaneal erosions where the 
Med Ultrason 2019; 21(4): 405-413

Table II. US and MRI concordances and discordances regarding ankle and hindfoot joints modifications

\begin{tabular}{lllllllllll}
\hline Joint & Parameter & MRI* & US $^{*}$ & MRI only* & US only* & PA & k & p-value & Se & Sp \\
\hline TT & Synovitis & 26 & 24 & 5 & 3 & 77.14 & $\mathbf{0 . 4 4 2}$ & $\mathbf{0 . 0 0 8}$ & 80.8 & 66.7 \\
& Erosions & 16 & 1 & 15 & 0 & 57.14 & 0.067 & 0.269 & 6.3 & 100.0 \\
& Osteophytes & 8 & 3 & 6 & 1 & 80.00 & 0.371 & 0.059 & 25.0 & 96.3 \\
TN & Synovitis & 23 & 21 & 5 & 3 & 77.14 & $\mathbf{0 . 5 1 2}$ & $\mathbf{0 . 0 0 2}$ & 78.3 & 75 \\
& Erosions & 12 & 5 & 9 & 2 & 68.57 & 0.189 & 0.191 & 25.0 & 91.3 \\
& Osteophytes & 8 & 7 & 5 & 4 & 74.29 & 0.237 & 0.159 & 37.5 & 85.2 \\
ST & Synovitis & 27 & 25 & 5 & 3 & 77.14 & $\mathbf{0 . 4 0 4}$ & $\mathbf{0 . 0 1 6}$ & 81.5 & 62.5 \\
& Erosions & 19 & 4 & 17 & 2 & 45.71 & 0.018 & 0.855 & 10.5 & 87.5 \\
& Osteophytes & 6 & 1 & 5 & 0 & 85.71 & $\mathbf{0 . 2 4 9}$ & $\mathbf{0 . 0 2 6}$ & 16.7 & 100.0 \\
\hline
\end{tabular}

*Data are presented as number of patients; MRI: magnetic resonance imaging; US: ultrasonography; PA: percent agreement; k: Cohen's kappa coefficient of agreement; Se: sensitivity; Sp: specificity; TT: tibiotalar joint; TN: talonavicular joint; ST: subtalar joint.

Table III. US and MRI concordances and discordances regarding ankle's tendon tenosynovitis

\begin{tabular}{|c|c|c|c|c|c|c|c|c|c|}
\hline Tendon & MRI* & US* & MRI only* & US only* & PA & $\mathbf{k}$ & p-value & Se & Sp \\
\hline $\mathrm{TA}$ & 7 & 4 & 3 & 0 & 91.14 & 0.681 & $<0.001$ & 100.0 & 57.1 \\
\hline EHL & 6 & 2 & 4 & 0 & 88.57 & 0.453 & 0.001 & 100.0 & 33.3 \\
\hline EDL & 2 & 1 & 1 & 0 & 97.14 & 0.653 & $<0.001$ & 100.0 & 50.0 \\
\hline $\mathrm{TP}$ & 9 & 7 & 3 & 1 & 88.57 & 0.677 & $<0.001$ & 96.2 & 66.7 \\
\hline FDL & 5 & 1 & 4 & 0 & 88.57 & 0.300 & 0.013 & 100.0 & 20.0 \\
\hline FHL & 6 & 1 & 6 & 1 & 80.00 & 0.052 & 0.644 & 96.6 & 0 \\
\hline Peroneal & 5 & 4 & 2 & 1 & 91.14 & 0.618 & $<0.001$ & 96.7 & 60.0 \\
\hline
\end{tabular}

*Data are presented as number of patients; MRI: magnetic resonance imaging; US: ultrasonography; PA: percent agreement; k: Cohen's kappa coefficient of agreement; Se: sensitivity; Sp: specificity; TA: tibialis anterior; EHL: extensor hallucis longus; EDL: extensor digitorum longus; TP: tibialis posterior; FDL: flexor digitorum longus; FHL: flexor hallucis longus.

Table IV. US and MRI concordances and discordances regarding heel's modifications

\begin{tabular}{|c|c|c|c|c|c|c|c|c|c|c|}
\hline Structure & Parameter & MRI* & US* & MRI only* & US only* & PA & $\mathbf{k}$ & p-value & Se & Sp \\
\hline \multirow[t]{4}{*}{ Achille's tendon } & Entesophytes & 19 & 28 & 2 & 11 & 62.86 & 0.217 & 0.127 & 31.3 & 89.5 \\
\hline & $\begin{array}{l}\text { Retrocalcaneal } \\
\text { bursitits }\end{array}$ & 20 & 10 & 13 & 3 & 54.29 & 0.138 & 0.331 & 80.0 & 35.0 \\
\hline & $\begin{array}{l}\text { Posterior calcaneal } \\
\text { erosions }\end{array}$ & 4 & 6 & 1 & 3 & 88.57 & 0.536 & 0.001 & 90.3 & 75.0 \\
\hline & Tendinosis & 7 & 4 & 4 & 2 & 80.00 & 0.255 & 0.111 & 92.9 & 28.6 \\
\hline \multirow[t]{3}{*}{ Plantar fascia } & $\begin{array}{l}\text { Thickness greater } \\
\text { than } 4 \mathrm{~mm}\end{array}$ & 3 & 22 & 0 & 19 & 45.71 & 0.105 & 0.164 & 40.6 & 100.0 \\
\hline & Perifasciitis & 7 & 8 & 4 & 5 & 74.29 & 0.237 & 0.159 & 82.1 & 42.9 \\
\hline & $\begin{array}{l}\text { Inferior calcaneal } \\
\text { erosions }\end{array}$ & 10 & 6 & 6 & 1 & 80.00 & 0.471 & 0.003 & 95.8 & 45.5 \\
\hline Subcalcaneal & & 9 & 8 & 1 & 0 & 97.15 & 0.928 & $<0.001$ & 96.2 & 100.0 \\
\hline
\end{tabular}

panniculitis

*Data are presented as number of patients; MRI: magnetic resonance imaging; US: ultrasonography; PA: percent agreement; k: Cohen's kappa coefficient of agreement; Se: sensitivity; Sp: specificity.

concordance was at least good) and heel's structure abnormalities.

We have two explanations for the superiority of MRI over US for synovitis evaluation in the ankle and hindfoot region found in our study. First comes from the MRI ability to visualize the entire joint and the deep part of the synovium, irrespective of the body fat tissue amount [26], while the US can visualize only the superficial joint recesses, not necessarily involved in cases of mild joint synovitis (as were the cases with synovitis not-identified by US). Second comes from the difficulty of US to differentiate synovial hypertrophy from fluid, especially in old, long-standing effusions, when fluid becomes hypoechoic in US scan [27]. Differentiation of fluid from synovitis can be done by applying compression with the transducer (synovial fluid is compressible and displaceable, while synovial hypertrophy is poorly compressible and nondisplaceable) and by the presence of the PD signal in the 


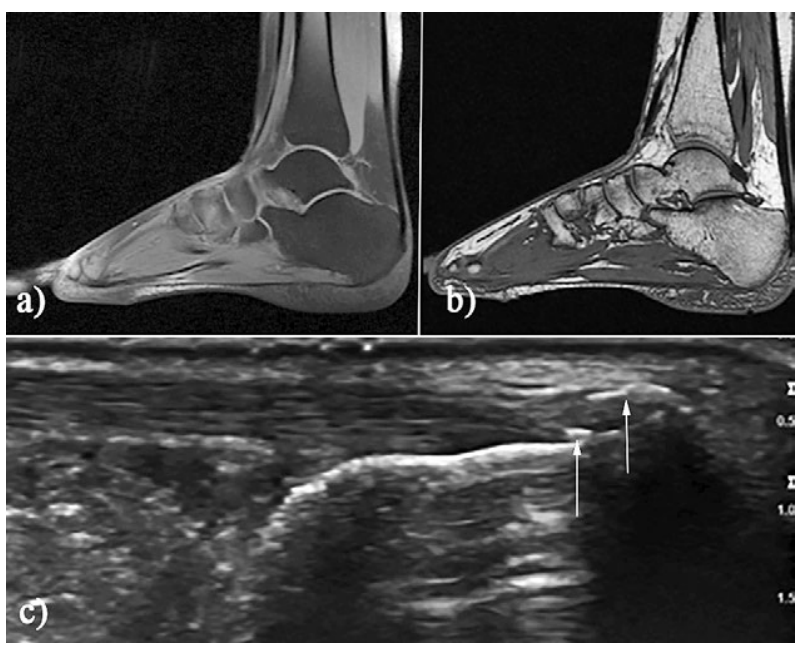

Fig 2. A 42-years old RA patients where MRI could not identify Achille's tendon enthesophytes in a) sagittal T1-weighted fat saturation (FS) and b) sagittal 3D T1 FSPGR. US identified two small enthesophytes (arrows) in c) longitudinal scan of the Achille's tendon.

synovial hypertrophy $[5,28]$. During the US examination of the ankle and hindfoot joints, the compression with the transducer is impossible and the PD sensitivity is lower in profound joints, especially in low activity synovitis. This can explain the misinterpretation by US of the synovial fluid as being synovial hypertrophy. In this regard, non-contrast MRI is also unable to differentiate between inflamed synovium and synovial fluid as both display the same signal intensity even in fluid-sensitive sequences. Only the use of contrast-enhanced MRI can make the distinction - the inflamed synovium enhances after contrast injection [26]. However, gadolinium administration raises the cost and duration of the MRI examination and adds considerable discomfort to the patients [29].

It was demonstrated that US and MRI are useful and reliable imaging techniques in identifying tenosynovitis $[5,12]$. As we expected, we found very good concordance between US and MRI in identifying the ankle's tenosynovitis. Analyzing the discordant cases, we observed that in some cases the US showed normal aspect and MRI found tenosynovitis. The presence of normal synovial fluid within the tendons sheath was demonstrated using MRI over 25 years ago [25], but for ankle tendons the subject was not developed until later. Willekens et al [30] evaluated the normal distribution of fluid within the tendon sheath of the ankle using MRI and found fluid in the retromalleolar or inframalleolar part of the tendons in healthy volunteers (apart from the anterior tendons), most commonly in medial tendons (the study that was published after our study was finished and we could not take into consideration their results for our MRI interpre-

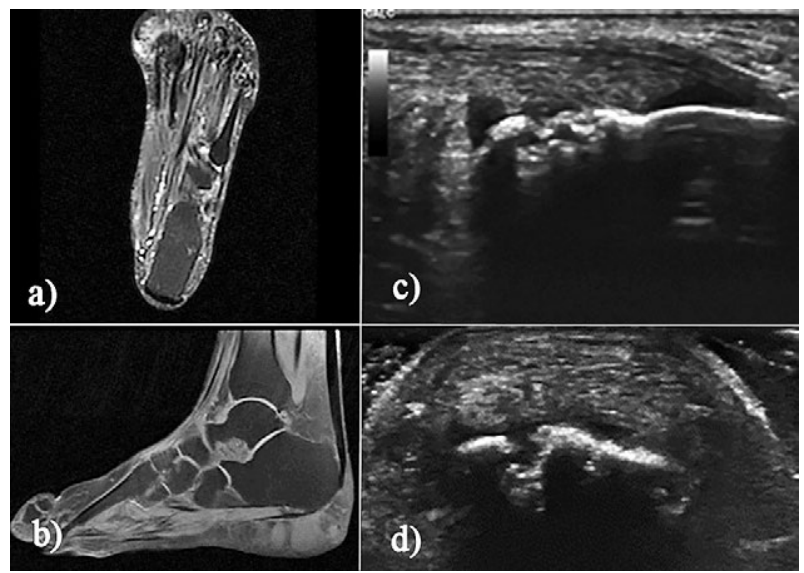

Fig 3. A 44-years old RA patient where MRI could not identify the retrocalcaneal bursitis and calcaneal erosions in a) axial STIR sequence and b) sagittal T1-weighted fat saturation (FS); c) longitudinal and d) transverse US of the Achille's tendon showed retrocalcaneal bursitis and calcaneal erosions.

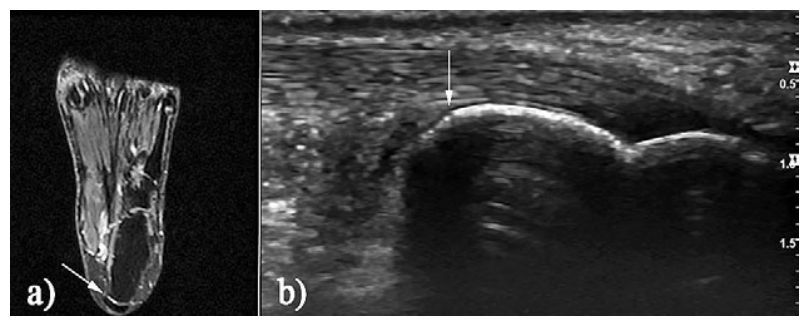

Fig 4. A 64-years old RA patient where MRI showed small retrocalcaneal bursitis (arrow) in a) axial STIR sequence and US showed normal aspect of retrocalcaneal bursa as a thin anechoic line (arrow) in b) longitudinal scan of the Achille's tendon.

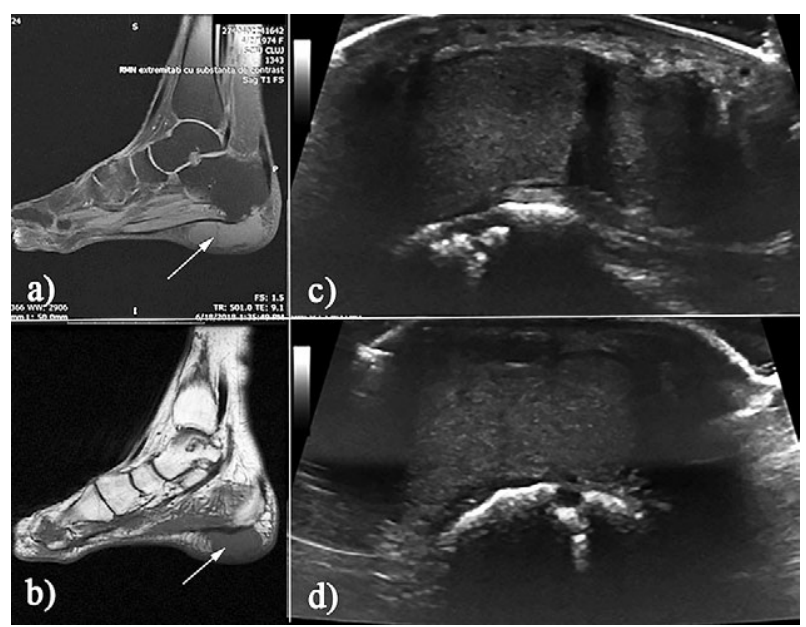

Fig 5. A 45-years old RA patient with subcalcaneal panniculitis and calcaneal erosions on MRI sequences a) sagittal T1-weighted fat saturation (FS) and b) sagittal T1-weighted fast spin echo (FSE) (arrows); c) longitudinal and d) transverse US scan of the plantar aspect of the heel showing the subcalcaneal panniculitis and calcaneal erosions. 
tation). For US examination, Schmidt et al [31] published in 2004 the reference values for musculoskeletal US in healthy subjects, to prevent misinterpretation. Over time, the subject regarding musculoskeletal US in healthy/ asymptomatic people was largely discussed [32-34], but a clear cut-off between normal and abnormal quantity of fluid in the ankle tendon sheath was not established. But, if in US examination a small quantity of fluid is found only under the level of malleoli, in the lower part of the tendon sheath, frequently not surrounding the tendon, the finding is interpreted as normal fluid, found here due to patient's position (the knee flexed $90^{\circ}$ and the sole resting on the bed) and gravity. In MRI examination, the fluid identified in 3 consecutive axial slices is interpreted as tenosynovitis [12]. But our radiologists did not take into consideration that the patient's position with the knee extended, the ankle flexed $90^{\circ}$ and the forefoot pointing upward produce a gravitationally movement of the normal fluid proximally and posteriorly and this can be seen on more than 3 consecutive slices as Schweitzer et al [35] demonstrated.

Bone marrow edema or osteitis (inflammation of the subchondral trabecular bone), findings specific for inflammatory arthropathy, denotes a severe and aggressive disease phenotype, and predicts the structural damage progression of the disease [29,36]. MRI is the only imaging technique capable of visualizing this abnormality.

The heel region, being superficial, can be easily evaluated by US, with comparable performance with MRI [37]. The thickness of the slices is an important factor for small abnormalities MRI visualization [38]. Comparing the MRI result obtained by using slices with $3 \mathrm{~mm}$ thickness with US results, we found that US found more enthesophytes and calcaneal erosions compared with MRI, similar with Aguila Maldonado et al [37] (thickness of slice not specified) and Baraliakos et al [39] (3-4 mm slice thickness) in spondylarthritis patients.

In our study, MRI found considerably more cases of retrocalcaneal bursitis than US. The bursitis definition in both imaging techniques imply the presence of bursal fluid collection, but using contrast-enhanced MRI, the inflammation is supplementary revealed. The retrocalcaneal bursitis could be accompanied by the inflammation of the surrounding tissues, especially the Kager's fat pad, that displays the same MRI findings as the retrocalcaneal bursitis, including the enhancement [40]. For this reason, the differentiation of retrocalcaneal bursitis from inflamed pre-Achilles fat pad can be difficult for MRI, while US can easily discriminate between the two situations.

We found significant more thickened plantar fascia using US compared with MRI, despite the fact that the measurements were performed at the same level, near the calcaneus insertion. Plantar fascia is a superficial structure, easily approachable by the US. Moreover, US being a real-time examination, it allows the lateral sweep of the transducer for identification of the thickest section through the plantar fascia. In MRI, the identification of the thickest sagittal section could be influenced by the thickness of the slices used for examination. Moraes do Carmo et al [41] performed a study on cadaveric specimens and compared the thickness of the plantar fascia using US, MRI and anatomic specimen. The authors found that both imaging techniques encountered a variability of the values, slightly higher for MRI.

Subcalcaneal panniculitis (or inflammatory-edematous lesion of the heel fat pad) is frequent in RA, more frequent than in spondylarthritis, and is associated with talalgia [42]. US and MRI are both capable of visualizing this lesion [19]. In our study, the agreement between US and MRI in identifying subcalcaneal panniculitis was very good.

The present study has some limitations. The small number of the patients included and the lack of comparison of RA patients US and MRI evaluations with healthy subjects are probably the most important limitations of our study. The main reason for these limitations is related of the high cost of the contrast-enhanced MRI. The use of a magnetic field strength of $1.5 \mathrm{~T}$ and the $3-\mathrm{mm}$ thickness of the slices contributed substantially to some of the encountered discordances. In some cases, the interpretation of the results was challenging for the radiologists due to the existence of movement artifacts in the patients that found it difficult to stand still for 40-45 minutes. Probably the use of dedicated musculoskeletal MRI machine could have avoided this limitation.

\section{Conclusion}

Both US and MRI are reliable and reproducible imaging techniques, with good and very good interobserver agreement for ankle, hindfoot and heel evaluation in RA patients. US has comparable performances with MRI for the identification of synovitis, tenosynovitis, erosions, osteophytes, retrocalcaneal bursitis and subcalcaneal panniculitis, having even more utility for the evaluation of enthesophytes and plantar fascia. The discordances between US and MRI could be explained in part by the equipment performances and settings and the diagnostic performances of the methods, but also by the different position of the lower limb during the examination, the different definitions for the same pathology, and the lack of consensus regarding the normality status.

Conflict of interest: None. 
Acknowledgements: The authors want to thank Dr. Ioana Felea and Dr. Laura Damian from the Rheumatology Department for their help. We acknowledge support from "Iuliu Hatieganu" University of Medicine and Pharmacy Cluj-Napoca, agreement nr. 3066/52/ 01.02.2018.

\section{References}

1. Colebatch AN, Edwards CJ, Ostergaard M, et al. EULAR recommendations for the use of imaging of the joints in the clinical management of rheumatoid arthritis. Ann Rheum Dis 2013;72:804-814.

2. Ogishima H, Tsuboi H, Umeda N, et al. Analysis of subclinical synovitis detected by ultrasonography and low-field magnetic resonance imaging in patients with rheumatoid arthritis. Mod Rheumatol 2014;24:60-68.

3. Rahmani M, Chegini H, Najafizadeh SR, Azimi M, Habibollahi P, Shakiba M. Detection of bone erosion in early rheumatoid arthritis: ultrasonography and conventional radiography versus non-contrast magnetic resonance imaging. Clin Rheumatol 2010;29:883-891.

4. Taniguchi D, Tokunaga D, Oda R, et al. Maximum intensity projection with magnetic resonance imaging for evaluating synovitis of the hand in rheumatoid arthritis: comparison with clinical and ultrasound findings. Clin Rheumatol 2014;33:911-917.

5. Wakefield RJ, Balint PV, Szkudlarek M, et al. Musculoskeletal ultrasound including definitions for ultrasonographic pathology. J Rheumatol 2005;32:2485-2487.

6. Szkudlarek M, Court-Payen M, Jacobsen S, Klarlund M, Thomsen HS, Ostergaard M. Interobserver agreement in ultrasonography of the finger and toe joints in rheumatoid arthritis. Arthritis Rheum. 2003;48:955-962.

7. Mathiessen A, Haugen IK, Slatkowsky-Christensen B, Boyesen P, Kvien TK, Hammer HB. Ultrasonographic assessment of osteophytes in 127 patients with hand osteoarthritis: exploring reliability and associations with MRI, radiographs and clinical joint findings. Ann Rheum Dis 2013;72:51-56.

8. D'Agostino MA, Terslev L, Aegerter P, et al. Scoring ultrasound synovitis in rheumatoid arthritis: a EULAR-OMERACT ultrasound taskforce-Part 1: definition and development of a standardised, consensus-based scoring system. RMD Open 2017;3:e000428.

9. Naredo E, D'Agostino MA, Wakefield RJ, et al. Reliability of a consensus-based ultrasound score for tenosynovitis in rheumatoid arthritis. Ann Rheum Dis 2013;72:1328-1334.

10. Balint PV, Terslev L, Aegerter P, et al. Reliability of a consensus-based ultrasound definition and scoring for enthesitis in spondyloarthritis and psoriatic arthritis: an OMERACT US initiative. Ann Rheum Dis 2018;77:1730-1735.

11. Moller I, Janta I, Backhaus M, et al. The 2017 EULAR standardised procedures for ultrasound imaging in rheumatology. Ann Rheum Dis 2017;76:1974-1979.

12. Ostergaard M, Peterfy CG, Bird P, et al. The OMERACT Rheumatoid Arthritis Magnetic Resonance Imaging
(MRI) Scoring System: Updated Recommendations by the OMERACT MRI in Arthritis Working Group. J Rheumatol 2017;44:1706-1712.

13. Ostergaard M, Peterfy C, Conaghan P, et al. OMERACT Rheumatoid Arthritis Magnetic Resonance Imaging Studies. Core set of MRI acquisitions, joint pathology definitions, and the OMERACT RA-MRI scoring system. J Rheumatol 2003;30:1385-1386.

14. Horikoshi M, Suzuki T, Sugihara M, et al. Comparison of low-field dedicated extremity magnetic resonance imaging with articular ultrasonography in patients with rheumatoid arthritis. Mod Rheumatol 2010;20:556-560.

15. Ohrndorf S, Boer AC, Boeters DM, et al. Do musculoskeletal ultrasound and magnetic resonance imaging identify synovitis and tenosynovitis at the same joints and tendons? A comparative study in early inflammatory arthritis and clinically suspect arthralgia. Arthritis Res Ther 2019; 21:59.

16. Enache L, Popescu CC, Micu M, et al. Ankle involvement in rheumatoid arthritis - a comparison of inflammatory signs on musculoskeletal ultrasound and magnetic resonance imaging. Med Ultrason 2019;21:265-272.

17. Lehtinen A, Paimela L, Kreula J, Leirisalo-Repo M, Taavitsainen M. Painful ankle region in rheumatoid arthritis. Analysis of soft-tissue changes with ultrasonography and MR imaging. Acta Radiol 1996;37:572-577.

18. Wakefield RJ, Freeston JE, O'Connor P, et al. The optimal assessment of the rheumatoid arthritis hindfoot: a comparative study of clinical examination, ultrasound and high field MRI. Ann Rheum Dis 2008;67:1678-1682.

19. Falsetti P, Frediani B, Acciai C, et al. Ultrasonography and magnetic resonance imaging of heel fat pad inflammatoryoedematous lesions in rheumatoid arthritis. Scand J Rheumatol 2006;35:454-458.

20. Wickman AM, Pinzur MS, Kadanoff R, Juknelis D. Healthrelated quality of life for patients with rheumatoid arthritis foot involvement. Foot Ankle Int 2004;25:19-26.

21. Aletaha D, Neogi T, Silman AJ, et al. 2010 Rheumatoid arthritis classification criteria: an American College of Rheumatology/European League Against Rheumatism collaborative initiative. Arthritis Rheum 2010;62:2569-2581.

22. Iagnocco A, Naredo E. Osteoarthritis: research update and clinical applications. Rheumatology (Oxford). 2012;51 Suppl 7:vii2-vii5.

23. Terslev L, Naredo E, Iagnocco A, et al. Defining enthesitis in spondyloarthritis by ultrasound: results of a Delphi process and of a reliability reading exercise. Arthritis Care Res (Hoboken) 2014;66:741-748.

24. Ostergaard M, Edmonds J, McQueen F, et al. An introduction to the EULAR-OMERACT rheumatoid arthritis MRI reference image atlas. Ann Rheum Dis 2005;64 Suppl 1:i3-i7.

25. Haugen IK, Lillegraven S, Slatkowsky-Christensen B, et al. Hand osteoarthritis and MRI: development and first validation step of the proposed Oslo Hand Osteoarthritis MRI score. Ann Rheum Dis 2011;70:1033-1038.

26. Burke CJ, Alizai H, Beltran LS, Regatte RR. MRI of synovitis and joint fluid. J Magn Reson Imaging 2019;49:15121527. 
27. Kane D, Grassi W, Sturrock R, Balint PV. Musculoskeletal ultrasound--a state of the art review in rheumatology. Part 2: Clinical indications for musculoskeletal ultrasound in rheumatology. Rheumatology (Oxford) 2004;43:829-838.

28. Terslev L. Synovitis. In: Wakefield R, D’Agostino M, editors. Essential Applications of Musculoskeletal Ultrasound in Rheumatology: Saunders Elsevier; 2010:67-77.

29. Rubin DA. MRI and ultrasound of the hands and wrists in rheumatoid arthritis. I. Imaging findings. Skeletal Radiol 2019;48:677-695.

30. Willekens I, Shahabpour M, Lenchik L, et al. Fluid distribution in ankle tendon sheaths in healthy volunteers: MRI findings. Surg Radiol Anat 2019 Oct 19. doi:10.1007/ s00276-019-02355-z.

31. Schmidt WA, Schmidt H, Schicke B, Gromnica-Ihle E. Standard reference values for musculoskeletal ultrasonography. Ann Rheum Dis 2004;63:988-994.

32. Nazarian LN, Rawool NM, Martin CE, Schweitzer ME. Synovial fluid in the hindfoot and ankle: detection of amount and distribution with US. Radiology 1995;197:275-278.

33. O'Connor PJ, Grainger AJ, Morgan SR, Smith KL, Waterton JC, Nash AF. Ultrasound assessment of tendons in asymptomatic volunteers: a study of reproducibility. Eur Radiol 2004;14:1968-1973.

34. Premkumar A, Perry MB, Dwyer AJ, et al. Sonography and MR imaging of posterior tibial tendinopathy. AJR Am J Roentgenol 2002;178:223-232.

35. Schweitzer ME, van Leersum M, Ehrlich SS, Wapner K. Fluid in normal and abnormal ankle joints: amount and distribution as seen on MR images. AJR Am J Roentgenol 1994;162:111-114.
36. Haavardsholm EA, Boyesen P, Ostergaard M, Schildvold A, Kvien TK. Magnetic resonance imaging findings in 84 patients with early rheumatoid arthritis: bone marrow oedema predicts erosive progression. Ann Rheum Dis 2008;67:794-800.

37. Aguila Maldonado R, Ruta S, Valuntas ML, Garcia M. Ultrasonography assessment of heel entheses in patients with spondyloarthritis: a comparative study with magnetic resonance imaging and conventional radiography. Clin Rheumatol 2017;36:1811-1817.

38. McQueen F, Ostergaard M, Peterfy C, et al. Pitfalls in scoring MR images of rheumatoid arthritis wrist and metacarpophalangeal joints. Ann Rheum Dis 2005;64 Suppl 1:i48i55.

39. Baraliakos X, Kiltz U, Appel H, et al. Chronic but not inflammatory changes at the Achilles' tendon differentiate patients with peripheral spondyloarthritis from other diagnoses - Results from a prospective clinical trial. RMD Open 2017;3:e000541.

40. Boutry N, Flipo RM, Cotten A. MR imaging appearance of rheumatoid arthritis in the foot. Semin Musculoskelet Radiol 2005;9:199-209.

41. Moraes do Carmo CC, Fonseca de Almeida Melao LI, Valle de Lemos Weber MF, Trudell D, Resnick D. Anatomical features of plantar aponeurosis: cadaveric study using ultrasonography and magnetic resonance imaging. Skeletal Radiol 2008;37:929-935.

42. Falsetti P, Frediani B, Acciai C, Baldi F, Filippou G, Marcolongo R. Heel fat pad involvement in rheumatoid arthritis and in spondyloarthropathies: an ultrasonographic study. Scand J Rheumatol 2004;33:327-331. 